Monatsschrift für Geburtshülfe und Gynaekologie 1896;3:81-84

\title{
Tages-Nachrichten und Notizen
}

Das Statut des St. Petersburger Medicinischen Frauen-Instituts.

Schon ei $\pi$ nial hat in Russland eine Art „Medicinisches Frauen-Institut” bestanden in den am Nicolai-Kriegshospital eingerichteten weiblichen Aerztekursen, die im Jahre 1882 aufgehoben wurden, trotzdem sie damals über 400 Hörerinnen zählten, welche sich nun nach dem Auslande, vorwiegend nach der Schweiz und nach Paris, wandten.

Zur Zeit sind in Russland noch 554 weibliche Aerzte thätig, die zum grössten Teil ihr Studium noch am Nicolai-Kriegshospital vollendet haben.

Wiederholte Gesuche, den weiblichen Studierenden die samtlichen russischen Fakultäten zu öffnen, lehnte die Regierung ab, um nun ein eigenes abgeschlossenes „Medicinisches FrauenInstitut und Internat" ins Leben zu rufen, welches von privater Seite vorbereitet und dotiert, sich ohne Staatszuschiisse erhalten soil. dtû 1. Januar 1897 soil es eröffnet werden.

In der Voraussetzung, dass diese Gründung. so verschieden sie von west-lichen Verhältnissen liegt, auch in Deutschland mit Interesse verfolgt werde, haben wir uns an Herrn Prof. v. Ott in St. Petersburg gewandt mit der Bitte, uns ein Exemplar des Statute verschafl $1 / 8 \mathrm{n}$ zu wollen.

Monatsschrift fur Geburtshülfe und Gynäkologie. 1896. Bd. III. Heft 1.6 82

Tagesnachrichten.

Für die freundlíche Bereitwilligkeit, mit welcher er unserer Bitte ent-عprochen hat, sagen wir ihra an dieser Stelle unseren verbindlichsten Dank.

1. Das Medicinische Fraueninstitut zu St. Petersburg ist gegründet, urn denFrauen eine medicinische Ausbildung zu verschaffen, hauptsächlich. zur Behandlung von Kindern und Frauen, wie auch zur geburtshülflichen Thätigkeit. Im Institut wird ein Jnternat eingerichtet für Zuhörermnen, die aicht bei ihren Eltern oder anderen zuverlässigen Personen wohnen können. Die Mittel de^. Institute sind folgende:

Zinsen von den zur Brrichtung des Institute gespendeten Summen.

Beiträge, die zur Unterhaltung des In\&tituts gespendet werden.

Zuschüsse.

Gebiihrengelder.

Das Institut ist dem Minister der Volksaufklärung, sowie auch dera Pro-kurator des St.

Petersburger Bezirks untergeordnet. Unmittelbar wird das Institut vom Direktor unter Beistand des Professorenrats, einer Direktion, eines Verwaltungsrates, sowie einer Inspection samt Gehülfinnen geleitet. Zum Direktor wird vom Minister ein erfahrener Professor der Medizin erwählt. Der Direktox ist zugleich Präsident des Professorenrats, der Direktion und des Verwaltungsrates. Die Direktion besteht aus dem Direktor, 4 vom Prokurator bestimmten Professoren, 2 vom Verwaltungsrat gewählten und vom Prokurator bestätigten Mítgliedern, sowie der Inspektrice. Die Direktion verhandelt über die wichtigsten wirtschaft-lichen, administrativen und disciplinären Fragen. Der Verwaltungsrat soigt für das materielle Gedeihen des Instituts. Zu dessen Mitgliedern werden vom Minister Personen ernannt, die zur 
Vergrösserung der Geldmittel des Instituts und zur Beförderung seiner wirtschaftlichen Angelegenheiten beitragen können.

Die unmittelbare Aufsicht über die Zuhörerinnen führt die Inspektrice sowie die Verwaltung des Internats. Die Inspektrice wird vom Direktor gewählt und auf Vorschlag des Prokurators vom Minister bestätigt. Die Gehülfinnen der Inspektrice sind ihr untergeordnet, werden von ihr erwählt und vom Prokurator auf Vorschlag des Direktors bestätigt. Die theoretischen und praktischen Studien werden von Personen geleitet, die bei einer medicinischen Fakultät das Jus docendi erlangt haben. Die Leitung der klinischen Studien wird vorzugsweise Direktoren und Oberärzten von Krankenhäusern übertragen. Erledigte Stellen werden vom Minister durch dazu berechtigte Personen besetzt, oder dieselben werden vom Direktor gewählt und auf Vorschlag des Prokurators vom Minister bestätigt. Als Zuhörerinnen werden Personen christlichen Glaubens aufgenommen im Alter von 20 bis 35 Jahren. In Ausnahmefällen können mit Zustimmung des Prokurators auch Personen von höherem Alter aufgenommen werden. Personen, die sich als Zuhörerinnen melden, haben dem Direktor ein Gesuch einzureichen unter Beilage folgender Dokumente: a) Zeugnis über das Alter, den Stand und Glauben; b) Certificat über die Beendigung der Studien an einer der erforderlichen Lehr-anstaiten; c) Polizeiliches Zuverlässigkeitsattest; d) Schriftliche Erlaubnis der Eltern oder, falls dieselben nicht mehr am Leben sind und die betreffende Zu-hörerin noch nicht 21 Jahre alt ist, die des Vormunds; bei Verheirateten ist die schriftliche Erlaubnis des Gatten erforderlich. Personen, die ein Gymnasium oder eine ihm gleichwertige Lehranstalt durchgemacht haben, werden in der lateinischen Sprache gemäss dem zum Eintritt in die Universität aufgestellten Programm examiniert. Personen, welche die höheren Frauenkurse beendigt oder von einem dazu berechtigten Gymnasium das Reifezeugnis erhalten haben, werden ohne Nachprüfung in das Institut aufgenommen. Der Betrag des Inscriptionsgeldes wird vom Minister nach dem Vorschlag des Verwaltungsrates bestimmt.

Im Institut und bei den praktischen Uebungen müssen die Zuhörerinnen eine Uniform tragen. Im Institut werden folgende Gegenstände vorgetragen:

Anatomie.

Normale Histologie und Embryologie.

Physiologie.

Allgemeine Pathologie.

Specielle Pathologie und Therapie, Medicinische Diagnostik und physio-logische Chemie.

Organische und anorsranische Chemie.

Physik.

Tagesnachrichten. 83

Mineralogie, Botaník und Zoologie (mit vergleichender Anatomie).

Pharmakognosie und Pharmacie.

Pharmakologie, Toxikologie, Balneo- und Hydro-Therapie.

Pathologische Anatomie und Histologie.

Geburtshülfe (Klinik). lá) Gynaekologie (Klinik).

Paediatrie (Klinik).

Hygiene.

Chirurgie und Desmurgie.

Operative Geburtshülfe (Klinik). 
Syphilidologie und Dermatologie (Klinik).

Ophthalmologie (Klinik).

Nerven- und Geisteskrankheiten (Klinik).

Falls genügende Mittel vorhanden sein werden, wird auch für Ohren-, Nasen- und

Kehlkopfkrankheiten ein besonderer Lehrstuhl eingerichtet werden. Der Studienplan, welcher die Verteilung der theoretischen Vorträge unter den Professoren, sowie die Organisation des klinischen Unterrichts regelt, wird im Professorenrat entworfen und vom Minister bestätigt. Die Studienzeit dauert 5 Jahre oder 10 Semester, von denen 4 Jahre auf die Ausbildung im Institut entfallen, während das 5. Jahr zu praktischen Uebungen in einem Kinder- oder Frauenkrankenhaus oder einer Gebäranstalt unter der L·eitung erfahrener Aerzte verwendet wird. Diejenigen Zuhörerinnen, welche in diesem Jahre ungenügende Fortschritte in den praktischen Uebungen zeigten, können noch auf ein oder zwei Jahre in den Krankenhäusern zuriickgehalten werden, Die Prüfungen werden in derselben Art wie an den medicinischen Fakultäten abgelegt. Der Vorsitzende und die Mitglieder der Kommission für das Staatsexamen werden vom Minister ernannt. Die Zuhörerinnen, welche ihr Examen gut bestanden haben, erhalten ein Diplom als „Frauen-Arzt”. Dasselbe giebt ihnen folgende Rechte: 1. Das Recht der ärztlichen Praxis im ganzen Reiche; 2) das Recht, aus den Apotbeken stark wirkende Mittel zu verschreiben; 3) das Recht, als Specíalistinnen für Kinder- und Frauenkrankheiten Stellen zu bekleiden in alien weiblichen Lehr-anstalten, in Kinder- und Frauenkrankenhäusern, in Diakonissenhäusern und an polizeilich-sanitären Instituten, jedoch ohne die Rechte des Staatsdienstes zu erhalten; 4) das Recht, auf dem Lande allgemeine Krankenhäuser zu verwalten und Stellen von Landärzten zu bekleiden, sowie auch in Städten Kinder-und Frauen - Abteilungen in allgenieinen Krankenhäusern zu leiten und im ganzen Kraiikenhause zu dejourieren; 5) das Recht, als Gehülfinnen des Gerichtsarztes bei medicinisch-gerichtlichen Untersuchungen von Frauen und Kindern zu funktionieren. Dagegen dürfen die Frauen-Aerzte nicht in Städten allgemeine Krankenhäuser noch männliche Abteilungen verwalten; sie dürfen nicht in den Kommissionen zur Untersuchung von Wehrpflichtigen mitwirken, noch selbstständig in gerichtlichmedicinischen Fragen als Sachverständige auftreten. Am Institut kann unter Binverständnis des Ministers der Volksauf klärung und ades Innern auch eine Abteilung zur Vorbereitung der Frauen für die pharma-ceutische Thätigkeit eingerichtet werden.

Robert B. Battey $\uparrow$.

Robert B. Battey, geboren am 26. November 1828 zu Richmond, ist am 8. November 1895 zu Rome im Staate Georgia, Vereinigte Staaten, gestorben.

Er lebte dort seit 1847 mit Ausnahme der Jahre 1872 - 1875, wo er als Professor der Geburtshülfe und Gynäkologie am „Atalanta medical College” wirkte und das „Atalanta medical and Surgical Journal" herausgab, in welchem auch diejenige Arbeit erschienen ist, welche ihn überall hin bekannt gemacht hat.

Es gebührt Battey mit Hegar und Laws on Tait der Ruhm, der dritte der Zeit seiner ersten Operation nach, selbstständig und gestützt auf wissen-schaftliche Begründung, die Castration, von ihm selbst anfänglich als ,normal Ovariotomy” bezeichnet, eingeführt zu haben. Hegar operierte zum ersten Male am 27. Juli 1872, Lawson Tait am 1. August 1872 und Battey am 17. August 1872. Wenn die amenkanischen

$6^{*}$

84

Litteratur -Verzeichnis. 
Fachgenossen, statt einer neutralen Benennung, vielfach noch von „Battey’s Operation” sprechen, obwohl dieselbe Art und Ziel wesentlich geändert hat, so mag dies immerhin gerechtfertigt sein. Battey vollíührte die Operation auch von der Soheide bezw. dem Douglas aus und ist darait auch der Begründer der vaginalen Oophorectomie gewesen.

Gerade He gar giebt in seiner ersten Veröffentlichung (Centralbl. f. Gyn. 1878, No. 2) sowie in seiner bald darauf erschienenen klassisehen Hauptarbeit (Sammlg. klin. Vortr. 135-138, 1878) eine Uebersicht und Kritik der bis dahin bekannten Fälle von Oophorectomie, besonders derjenigen von Battey, dem gegenüber er einfach die Thatsache hervorhebt, vor ihm operiert zu haben, während allerdings Battey seine Operation bereits 1872 bekann $1 / 8$ nachte.

Der Hauptthat seines Lebens widmete Battey später noch mehrere Schriften. In einer von der Redaktion des Amer. Journ. of Med. Sciences 1886 veranlassten Erörterung über die Castration bei Gemüts- und Nervenkrankheiten sehen wir ihn neben Hegar und Spencer Wells sein Urteil abgeben und sich jetzt dagegen verwahren, dass er normale Ovarien entferne.

Auf der 12. Versammlung der Amer. Gynäc. Soc. in New-York, 1887 berichtete er über seine eigene Casuistik von 54 Fallen von „Battey’s Operation”, wie er sie nun selbst bezeichnet, allerdings ein Beweis, dass er si $\theta$ nicht missbräuchlich oft angewendet hatte.

Die Herbeiführung der Menopause war ihm stets Hauptzweck der Operation, die er sicherlich in vielen Fallen unvollständig und gefolgt von Nach-erkrankungen ausführte: er würde sonst auch nicht selbst ehrlich bekannt haben, dass die nachfolgenden climacterischen Störungen bis zu 5 Jahren andauem könnten.

Wenn Battey demnach an der späteren Weiterentwicklung der Operation zur Entfernung der Adnexa ad hoc bei alien überhaupt vorkommenden schweren Erkrankungen und zum Teil mit dem Zweeke, die Menopause gerade nicht ein-treten zu lassen, weniger beteiligt ist, so bleibt ihm dennoch der Ruhm, diese gynäkologische Hauptoperation mit inauguriert zu haben, ungeschmälert für "lie Zeiten.

G. H. van der Meij †.

G. H. van der Meij wurde geboren am 27. Januar 1851. Nacbdem er in Amsterdam seine Studien absolviert hatte, war er Assistent von Lehmann. Danach verbrachte er ungefähr ein Jahr in Wien, wo er bei Billroth und speciell bei Braun arbeitete. Nach seiner Rüc $\cdot k k e h r$ wurde er 1878 Privatdocent an der Universität und Lehrer der Hebammenschule in Amterdam. Nach Lehmann's Tode wurde er 1881 zu seinem Nachfolger ernannt. Obwohl er auch in der operativ $\beta n$ Gynäkologie bewandert und thätig war und sich zu einem sehr tüchtigen Gynäkologen ausgebildet hatte, so war van der Meij doch an erster Stelle Geburtshelfer. Verschiedene holländische Publikationen sind seiner Feder entsprungen - über das Mutterkorn, Uterusruptur, Sectio caesarea und neuerdings über Uteruskrebs bei Schwangerschaft bezeugen dies aufs glänzendste. Beim Kaiserschnitt besonders hatte er ausserordentlich günstige Resultate und stand in dieser Beziehung weitaus an der Spitze seiner Landsleute.

Am 7. Dezember zog er sich beim Durchstechen der Unterbindungsnadel während einer Pyosalpingotomie eine kleine Verwundung am Mittelfinger der linken Hand zu. Darauf folgte zwei Tage später, ohne ernste lokale Symptome, eine akute allgemeine Sepsis, der er am 16. Dezember erlag. Ehrenvoller Tod - aber um so schmerzlicheri Die holländische ärztliche Welt verliert in van der Meij einen geistvollen Kollegen und die'ihn gekannt haben, einen lieben Freund!

H. Treub.

Personalien.

Privatdocent Dr. Dührssen ist das Prädicat Professor verliehen worden. - 